\title{
Relationship between probable sarcopenia and nutritional disorders as evaluated using the Geriatric Nutritional Risk Index and serum phosphorus concentration in maintenance dialysis patients: A cross-sectional study
}

Kenichi KONO ( $\square$ kohno1209@gmail.com )

International University of Health and Welfare

Yoshihumi MORIYAMA

Department Wellness center, Kaikoukai Nagoya Kyoritsu Hospital

Ayaka HARA

Dialysis division at Shizuoka, Kaishokai

Hiroki YABE

School of Rehabilitation, Seirei Christoper University

Yusuke NISHIDA

Department Physical Therapy, International University of Health and Welfare Graduate School

Tetsuya YAMADA

Dialysis division, Kaikoukai healthcare group

Research article

Keywords: sarcopenia, nutrition, Geriatric Nutritional Risk Index, phosphorus, malnutrition

Posted Date: November 11th, 2019

DOI: https://doi.org/10.21203/rs.2.17120/v1

License: (c) (i) This work is licensed under a Creative Commons Attribution 4.0 International License.

Read Full License 


\section{Abstract}

Background To explore the relationship between sarcopenia and nutritional disorders, as evaluated using the Geriatric Nutritional Risk Index (GNRI) and serum phosphorus concentration (P).

Methods: This cross-sectional study analyzed 909 Japanese maintenance hemodialysis patients from a multicenter hemodialysis unit. The nutritional indices (GNRI and P) and sarcopenia indices [grip strength and Short Physical Performance Battery (SPPB)] were evaluated in all patients. Multiple logistic regression analysis was performed with the sarcopenia indices as dependent variables and GNRI only, $P$ only, and combined GNRI and P as independent variables adjusted for patient attributes. The odds ratio $(\mathrm{OR})$ and $95 \%$ confidence interval $(95 \% \mathrm{Cl})$ for the reference value were calculated by setting the category reference value to $\mathrm{GNRI} \geq 90$ and $\mathrm{P}$ to $3.6-5.0 \mathrm{mg} / \mathrm{dL}$. The main outcome measure was presence of probable sarcopenia.

Results: Grip strength was associated with decreased GNRI only (OR: 2.27, 95\% Cl: 1.64-3.16) and decreased GNRI and P (OR: 3.77, 95\% Cl: 1.49-9.53). In addition, SPPB was associated with decreased GNRI only (OR: 1.78, 95\% Cl: 1.30-2.44) and decreased GNRI and P (OR: 8.82, 95\% Cl: 3.52-22.12).

Conclusions: Nutritional disorders, in which both GNRI and P are decreased, are strongly related to sarcopenia compared with nutritional disorders in which only GNRI or only P is decreased.

\section{Background}

Protein energy wasting syndrome (PEW), which causes adverse changes in nutrition and body composition, is highly prevalent in patients with chronic kidney disease, especially in those undergoing dialysis, and is associated with high morbidity and mortality. ${ }^{1}$ It has been reported that PEW is related to appetite ratings. ${ }^{2}$ In addition, it has been pointed out that malnutrition accelerates aortic calcification and is closely related to cardiovascular disease onset and mortality. ${ }^{3,4}$ In these studies, nutritional status was assessed using the Geriatric Nutritional Risk Index (GNRI). GNRI is used to assess serum albumin kinetics and physical condition and has been utilized as a nutritional assessment index in Japanese dialysis patients; the clinical usefulness of GNRI has also been reported. . $^{3,5,6}$

Moreover, serum phosphorus concentration $(P)$ is used as another clinical evaluation index to assess the nutritional status of patients undergoing dialysis treatment. $\mathrm{P}$ is influenced by phosphorus intake and absorption. Therefore, as the specificity of malnutrition assessment is considered to be different between $P$ and GNRI, it is necessary to investigate these two indicators in order to know their impact on epidemic sarcopenia. A previous study on the relationship between GNRI and P and mortality showed that higher GNRI and lower $P$ were not significantly associated with patient mortality. ${ }^{7}$ On the other hand, even if $P$ is high or low, when GNRI is low, the mortality rate is significantly high. ${ }^{7}$ In addition, malnutrition is reported

to lead not only to death but also to sarcopenia. ${ }^{8}$ Therefore, it is clinically useful to clarify the relationship between sarcopenia and nutritional status evaluated by GNRI and P. However, only a few studies have 
evaluated nutritional status by combining GNRI and P and investigated the relationship between GNRI and $\mathrm{P}$ and sarcopenia.

We hypothesized that nutritional disorders can be likely more detected when evaluating GNRI and $P$ together rather than when evaluating GNRI and P separately. The purpose of this study was to explore the relationship between sarcopenia and nutritional disorders evaluated using GNRI and P in patients undergoing maintenance dialysis.

\section{Methods}

\section{Study population and design}

This cross-sectional study included clinically stable Japanese outpatients in multicenter hemodialysis unit from April 2012 to April 2018. Exclusion criteria included age $<18$ years, dialysis vintage $<6$ months, and refusal to participate. This study was approved by the Ethical Committee of International University of Health and Welfare (Approval number. 17-lo-95).

\section{Demographic and clinical laboratory findings}

Patients' demographics, such as age, dialysis vintage, and body mass index (BMI) were investigated. Laboratory values of serum albumin, serum hemoglobin, $C$ reactive protein, serum intact parathyroid hormone, standardized dialysis volume (Kt/V), and normalized protein catabolic rate (nPCR) were also collected.

\section{Assessment of nutritional status and classification of malnutrition by GNRI and P}

GNRI was calculated using the formula described elsewhere: $G N R I=[1.489 x$ serum albumin $(\mathrm{g} / \mathrm{L})]+$ [41.7x(body weight/ideal body weight)]., Ideal body weight was defined as weight with a BMI value of $22 \mathrm{~kg} / \mathrm{m}^{2} .{ }^{10}$ Malnutrition was defined as a GNRI $<90$ according to previous studies. ${ }^{3,11}$

$P$ was classified into three categories according to the guidelines of the Japanese Society of Dialysis Therapy ${ }^{12}$ : a $P$ of $3.5-6.0 \mathrm{mg} / \mathrm{dL}$ was considered the reference range, $\mathrm{P}<3.5 \mathrm{mg} / \mathrm{dL}$ was considered decreased, and $\mathrm{P}>6.0 \mathrm{mg} / \mathrm{dL}$ was considered abnormally high. ${ }^{12}$

In addition, GNRI and P were categorized together as follows: the reference category was set as GNRI $\geq 90$ and $3.5 \leq \mathrm{P} \leq 6.0$, whereas six other categories, including $2 \mathrm{GNRI}$ categories and $3 \mathrm{P}$ categories were also formed. 


\section{Measurement of indicators related to sarcopenia}

The diagnosis and definition of sarcopenia have been revised in $2018 .{ }^{13}$ In its 2018 definition, low muscle strength was pointed out as the primary parameter of sarcopenia; presently, muscle strength is considered the most reliable measure of muscle function. ${ }^{13}$ In addition, physical performance was formerly considered part of the core definition of sarcopenia. ${ }^{13}$ In the definition of 2018, physical performance was regarded as an index for determining severity. According to this new algorithm, ${ }^{13}$ we evaluated muscle strength by using grip strength test and physical performance by using Short Physical Performance Battery (SPPB) to determine probable sarcopenia. The cut-off value of grip strength was 26 $\mathrm{kg}$ for men and $18 \mathrm{~kg}$ for women from an Asian consensus ${ }^{14}$ and that of SPPB was 8 points. Grip strength was measured using a Smedley-spring type dynamometer (101A HATS, Tokyo).

\section{Statistics}

All values are expressed as mean \pm standard deviation (SD) or percentage, whenever appropriate. Descriptive statistics of nutrition and sarcopenia index were calculated by age group and were compared by one-way analysis of variance (ANOVA) and post hoc multiple comparisons. To analyze the relationship between probable sarcopenia and nutritional disorder, multiple logistic regression analyses were performed with grip strength and SPPB as dependent variables, and GNRI only, P only, and 6 categories of combined GNRI and P as independent variables. Results from logistic regression analyses were presented as odds ratios (OR) with $95 \%$ confidence intervals. A P value $<0.05$ was considered statistically significant. Statistical analyses were performed using SPSS (version 24, IBM, Tokyo).

\section{Results}

\section{Patient demographic, nutritional status, and sarcopenia index by age group}

A total of 1141 Japanese patients were registered. 232 patients in whom GNRI and P data could not be obtained were excluded. Finally, 909 patients were analyzed.

Table 1 shows the patient's demographic, nutritional status, and sarcopenia index.

Table 2 shows the nutritional status and sarcopenia index according to 5 age groups. The patients' mean $( \pm S D)$ age was $69.4( \pm 11.4)$ years. As shown in Table 2, 462 patients (56\%) were elderly (age $>70$ years). Regarding nutritional status, the mean GNRI was 91.5 , which decreased significantly with increasing age. In particular, the mean GNRI value in patients aged $>80$ years was 87.9 , indicating malnutrition. In addition, grip strength and SPPB scores as sarcopenia indices decreased significantly with increasing 
age. In elderly patients aged $\geq 80$ years, grip strength and SPPB scores were lower than the diagnostic criteria for probable sarcopenia.

\section{Relationship between nutritional status and grip strength as sarcopenia indices}

Table 3 shows the relationship between GNRI and P (nutritional indices) and grip strength (probable sarcopenia index). When analyzing GNRI and P separately, the GNRI of $<90$ showed a significant association with grip strength as a probable sarcopenia index (OR 2.272, 95\% Cl 1.636-3.155), but P did not show a significant association with grip strength. Furthermore, when GNRI and P were analyzed together, grip strength was significantly lower regardless of $\mathrm{P}$ in $\mathrm{GNRI}<90$. In particular, when $\mathrm{P}$ was $<3.5$ $\mathrm{mg} / \mathrm{dL}$, the OR of the decreasing grip strength as probable sarcopenia was 3.77 (95\% $\mathrm{Cl} 1.491-9.533$ ).

\section{Relationship between nutritional status and SPPB as sarcopenia indices}

Table 3 shows the relationship between GNRI and P (nutritional indices) and SPPB (probable sarcopenia index). When analyzing GNRI and P separately, the GNRI of $<90$ showed a significant association with SPPB (OR 1.782, 95\% Cl 1.303-2.437) as a probable sarcopenia index, but $P$ did not show a significant association with grip strength. Furthermore, when GNRI and $P$ were analyzed together, SPPB was significantly lower in $\mathrm{P}<6.0 \mathrm{mg} / \mathrm{dL}$ and $\mathrm{GNRI}<90$. In particular, when $\mathrm{P}<3.5 \mathrm{mg} / \mathrm{dL}$, the $\mathrm{OR}$ of the decreasing SPPB as a sarcopenia index was 6.77 (95\% Cl 2.658-17.243).

\section{Discussion}

In this observational study, we showed the relationship between sarcopenia and malnutrition status as evaluated by GNRI and P. It was revealed that both decreased GNRI and P lead to the presence of sarcopenia. When predicting sarcopenia using GNRI, obtained by evaluating the physique and serum albumin kinetics, and $\mathrm{P}$, patients with $\mathrm{GNRI}<90$ and $\mathrm{P}<3.5 \mathrm{mg} / \mathrm{dL}$ had the highest OR for lower grip strength and SPPB score.

Recently, GNRI has been used to screen the nutritional status of patients with various diseases. A report on the usefulness of GNRI in dialysis patients was published in 2008. ${ }^{5}$ In addition, it has been reported that GNRI has a higher sensitivity and specificity than the Mini Nutritional Assessment Short Form (MNASF) for predicting the cut-off value of the Malnutrition-Inflammation Score (MIS) ${ }^{5,15}$; the cut-off value was 91.2. ${ }^{5}$ Moreover, if the outcome is mortality, the cut-off value of GNRI has been reported to be 90.5 in Japanese dialysis patients. ${ }^{16}$ However, the value of GNRI for predicting sarcopenia has not been fully reported. Referring to the previous study investigating the relationship between sarcopenia and GNRI, 
muscle strength and muscle mass were found to be related to GNRI, ${ }^{17}$ and the cut-off value of GNRI for maintaining gait ability on their own was $86.7 .^{18}$ To estimate sarcopenia, we considered that the findings of the previous studies support the results in our present study, which are useful to investigate whether the value GNRI should be $<90$ in assessing nutritional status. Furthermore, in the analysis by age group, we should note that GNRI decreases along with increasing age; nearly half of all patients aged $>70$ years had GNRI of $<90$.

In addition, the effect of age was also observed in P. P in dialysis treatment is a general management index of chronic kidney disease mineral bone disorder (CKD-MBD). Hyperphosphatemia is a risk factor for ectopic calcification and mortality. ${ }^{19-21}$ Patients with hypophosphatemia, which is closely associated with restriction of protein intake, are known to have increased mortality risk. ${ }^{22,23}$ Recently, hemodialysis and medications were considered as management options for these patients to increase their protein intake. Moreover, the validity of $\mathrm{P}$ for assessing nutrition status has been reported. In our study findings, $\mathrm{P}$ $>3.5 \mathrm{mg} / \mathrm{dL}$ was shown to be not related to grip strength and SPPB in the adjusted model. It was indicated that assessing malnutrition using only $\mathrm{P}$ for predicting sarcopenia should be done cautiously. The absence of relationship between probable sarcopenia and $\mathrm{P}$, unlike GNRI, may be due to the fact that $P$ could be altered by medications based on the result of regular blood testing in a short period of time. In the management of CKD-MBD, it was suggested that $P$ should be strictly maintained within the normal range (3.5-6.0 mg/dL) compared to calcium concentration and that of intact parathyroid hormone. ${ }^{12}$ It has been reported that the phosphate binder improves the life prognosis ${ }^{24}$ of patients with $P>5.2$ $\mathrm{mg} / \mathrm{dL} .{ }^{25}$ However, in patients with $\mathrm{P}<3.7 \mathrm{mg} / \mathrm{dL}$ at baseline, it has been known that it is difficult to obtain the effect of improving life prognosis even if patients were treated by a phosphate binder. ${ }^{24}$ The reason why life prognosis is less likely to improve in patients with low phosphorus is considered to be due to the involvement of malnutrition. Therefore, it is important to evaluate protein uptake by GNRI as well as phosphorus.

This study has several limitations. First, we could not investigate detailed complications affecting feeding and metabolism and nutritional states, such as diabetes, stroke, and gastrointestinal cancer, as well as conduct an analysis adjusted for these factors. Second, we were not able to obtain detailed dosing data, such as phosphorus adsorbent and activated vitamin D preparation affecting serum phosphorus concentration. Moreover, the dialysis membranes affecting serum albumin kinetics were also not investigated. Finally, we could not show the causal association between aging, malnutrition, and probable sarcopenia, because the study had a retrospective cross-sectional design.

\section{Conclusion}

A nutritional disorders, presenting with a decreased in both GNRI and P, are strongly related to sarcopenia compared with nutrition disorders showing only abnormalities in only GNRI or only P. Multilateral observation of protein uptake kinetics, physique, and $\mathrm{P}$ is useful in predicting sarcopenia in dialysis patients. Our findings provide additional data for identifying patients with a high risk of sarcopenia using 
two nutritional indicators, such as GNRI and P. Moreover, these data will determine patients who require exercise interventions to reduce their sarcopenia risk. As part of the management of dialysis patients, it is necessary to recognize that their nutritional status and physical function decrease with age. When estimating the physical function deterioration related to sarcopenia by nutritional status, it is useful to not only analyze GNRI or phosphorus separately but also both indices together.

\section{Abbreviations}

GNRI: Geriatric Nutritional Risk Index; P: phosphorus; SPPB: Short Physical Performance Battery; OR: odds ratio; 95\% Cl: 95\% confidence interval; PEW: Protein energy wasting syndrome; BMl: body mass index; Kt/V; standardized dialysis volume; nPCR: normalized protein catabolic rate; SD: standard deviation; CKD-MBD: chronic kidney disease mineral bone disorder

\section{Declarations}

\section{Ethics approval and consent to participate}

The study protocol complied with the Helsinki Declaration standards and was approved by the Ethical Committee of International University of Health and Welfare (Approval number 17-lo-95). The requirement of written informed consent was waived as this study used retrospective data.

\section{Consent for publication}

Not Applicable.

\section{Availability of data and materials}

The datasets generated and/or analyzed during the current study are not publicly available but are available from the corresponding author on reasonable request.

\section{Competing interests}

The authors declare that they have no competing interest.

\section{Funding}

This work was supported by grants by the JSPS KAKENHI [Grant Number 17 K13097]. The funding source had no role in the design, data collection, analysis, and findings. 


\section{Authors' contributions}

$\mathrm{KK}, \mathrm{YM}, \mathrm{AH}, \mathrm{HY}, \mathrm{YN}$, and TY contributed to the conception and design of the work, the acquisition, analysis and interpretation of data, drafting or revision of the manuscript, and final approval of the version to be submitted.

\section{Acknowledgements}

The authors thank the renal staff for their support and the patients for giving their time to complete the research protocol.

\section{References}

1. Carrero JJ, Stenvinkel P, Cuppari L, Ikizler TA, Kalantar-Zadeh K, Kaysen G, et al. Etiology of the protein-energy wasting syndrome in chronic kidney disease: a consensus statement from the International Society of Renal Nutrition and Metabolism (ISRNM). J Ren Nutr. 2013;23:77-90.

2. Sahathevan $\mathrm{S}, \mathrm{Se} \mathrm{CH}, \mathrm{Ng} \mathrm{CH}$, Chinna K, Harvinder GS, Chee WS, et al. Assessing protein energy wasting in a Malaysian haemodialysis population using self-reported appetite rating: a crosssectional study. BMC Nephrol. 2015;16:99.

3. Okamoto T, Hatakeyama S, Kodama H, Horiguchi H, Kubota Y, Kido K, et al. The relationship between poor nutritional status and progression of aortic calcification in patients on maintenance hemodialysis. BMC Nephrol. 2018;19:71.

4. Panichi V, Cupisti A, Rosati A, Di Giorgio A, Scatena A, Menconi O, et al. Geriatric nutritional risk index is a strong predictor of mortality in hemodialysis patients: data from the Riscavid cohort. J Nephrol. 2014;27:193-201.

5. Yamada K, Furuya R, Takita T, Maruyama Y, Yamaguchi Y, Ohkawa S, et al. Simplified nutritional screening tools for patients on maintenance hemodialysis. Am J Clin Nutr. 2008;87:106-13.

6. Honda H, Qureshi AR, Axelsson J, Heimburger O, Suliman ME, Barany P, et al. Obese sarcopenia in patients with end-stage renal disease is associated with inflammation and increased mortality. $\mathrm{Am} \mathrm{J}$ Clin Nutr. 2007;86:633-8.

7. Fukuma S, Ikenoue T, Akizawa T, Fukuhara S. Impact of nutritional index on the association between phosphorus concentrations and mortality in haemodialysis patients: a cohort study from dialysis outcomes and practice pattern study in Japan. BMJ Open. 2017;7:e016682.

8. Cruz-Jentoft AJ, Baeyens JP, Bauer JM, Boirie Y, Cederholm T, Landi F, et al. Sarcopenia: European consensus on definition and diagnosis. Age Ageing. 2010;39:412-23.

9. Bouillanne O, Morineau G, Dupont C, Coulombel I, Vincent JP, Nicolis I, et al. Geriatric Nutritional Risk Index: a new index for evaluating at-risk elderly medical patients. Am J Clin Nutr. 2005;82:777-83. 
10. Shah B, Sucher K, Hollenbeck CB. Comparison of ideal body weight equations and published heightweight tables with body mass index tables for healthy adults in the United States. Nutr Clin Pract. 2006;21:312-9.

11. Kobayashi I, Ishimura E, Kato Y, Okuno S, Yamamoto T, Yamakawa T, et al. Geriatric Nutritional Risk Index, a simplified nutritional screening index, is a significant predictor of mortality in chronic dialysis patients. Nephrol Dial Transplant. 2010;25:3361-5.

12. Fukagawa M, Yokoyama K, Koiwa F, Taniguchi M, Shoji T, Kazama JJ, et al. Clinical practice guideline for the management of chronic kidney disease-mineral and bone disorder. Ther Apher Dial. 2013;17:247-88.

13. Cruz-Jentoft AJ, Bahat G, Bauer J, Boirie Y, Bruyère O, Cederholm T, et al. Sarcopenia: revised European consensus on definition and diagnosis. Age Ageing. 2019; 48:16-31.

14. Chen LK, Liu LK, Woo J, Assantachai P, Auyeung TW, Bahyah KS, et al. Sarcopenia in Asia: consensus report of the Asian Working Group for sarcopenia. J Am Med Dir Assoc. 2014;15:95-101.

15. Kalantar-Zadeh K, Kopple JD, Block G, Michael H. Humphreys. A malnutrition-inflammation score is correlated with morbidity and mortality in maintenance hemodialysis patients. Am J Kidney Dis. 2001;38:1251-63.

16. Fujii T, Hattori H, Nakahama K, Yamada Y. Prognostic expectation of hemodialysis patients using Geriatric Nutritional Risk Index (GNRI): comparison with single application of Body Mass Index (BMI) or serum albumin. Journal of Japanese Society for Dialysis Therapy. 2014;47:75-84.

17. Beberashvili I, Azar A, Sinuani I, Shapiro G, Feldman L, Sandbank J, et al. Geriatric Nutritional Risk Index, muscle function, quality of life and clinical outcome in hemodialysis patients. Clin Nutr. 2016;35:1522-9.

18. Tanaka T, Nishiyama K, Yamamura O, Watase H, Yokoyama $Y$, Horiguchi T, et al. Geriatric Nutritional Risk Index for independent walking function in maintenance hemodialysis patients: a single-facility retrospective cohort study. Geriatr Gerontol Int. 2018;18:1556-61.

19. Block GA, Klassen PS, Lazarus MJ, Ofsthun N, Lowrie EG, Chertow GM. Mineral metabolism, mortality, and morbidity in maintenance hemodialysis. J Am Soc Nephrol. 2004;15:2208-18.

20. Moe S, Drueke T, Cunningham J, Goodman W, Martin K, Olgaard K, et al. Definition, evaluation, and classification of renal osteodystrophy: a position statement from Kidney Disease: Improving Global Outcomes (KDIGO). Kidney Int. 2006;69:1945-53.

21. Chiroli S, Mattin C, Belozeroff V, Perrault L, Mitchell D, Gioni I. Impact of mineral and bone disorder on healthcare resource use and associated costs in the European Fresenius medical care dialysis population: a retrospective cohort study. BMC Nephrol. 2012;13:140.

22. Nakai S, Akiba T, Kazama J, Yokoyama K, Fukagawa M, Tominaga Y, et al. Effects of serum calcium, phosphorous, and intact parathyroid hormone levels on survival in chronic hemodialysis patients in Japan. Ther Apher Dial. 2008;12:49-54.

23. Shinaberger CS, Greenland S, Kopple JD, Van Wyck D, Mehrotra R, Kovesdy CP, et al. Is controlling phosphorus by decreasing dietary protein intake beneficial or harmful in persons with chronic kidney 
disease? Am J Clin Nutr. 2008;88:1511-8.

24. Isakova T, Gutierrez OM, Chang Y, et al. Phosphorus binders and survival on hemodialysis. J Am Soc Nephrol. 2009;20:388-96.

25. Fernández-Martín JL, Martínez-Camblor P, Dionisi MP, Floege J, Ketteler M, London G, et al. Improvement of mineral and bone metabolism markers is associated with better survival in haemodialysis patients: the COSMOS study. Nephrol Dial Transplant. 2015;30:1542-51.

\section{Tables}

Table 1. Patients' demographic data, nutritional status, and sarcopenia index

\begin{tabular}{lc}
\hline Age (years) & $69.4(11.4)$ \\
Sex (male/female) & 498 / 411 \\
Dialysis vintage (month) & $82.3(91.2)$ \\
BMI (kg/m²) & $22.0(4.22)$ \\
& $3.55(0.33)$ \\
Serum albumin (g/dL) & \\
GNRI & $91.5(6.56)$ \\
Serum hemoglobin (g/dL) & $10.9(1.07)$ \\
CRP (mg/dL) & $0.79(3.43)$ \\
\hline Serum intact-PTH (pg/dL) & $158.3(169.0)$ \\
\hline
\end{tabular}

GNRI, Geriatric Nutritional Risk Index; CRP, C-reactive protein; SPPB, Short Physical Performance Battery; PTH, parathyroid hormone; BMI, body mass index 
Table 2. Nutritional status and sarcopenia index by age group

\begin{tabular}{lccccccc}
\hline & $\leq 49$ years & $50-59$ years & $60-69$ years & $70-79$ years & $\geq 80$ & P value \\
& $(\mathrm{n}=60)$ & $(\mathrm{n}=88)$ & $(\mathrm{n}=249)$ & $(\mathrm{n}=350)$ & $(\mathrm{n}=162)$ & \\
\hline GNRI & $95.8(5.11)$ & $94.2(4.85)$ & $92.9(6.11)$ & $90.7(6.71)$ & $87.9(6.05)$ & $<0.01$ \\
Serum phosphorus (mg/dL) & $5.76(1.44)$ & $5.43(1.35)$ & $5.26(1.27)$ & $5.04(1.17)$ & $4.84(1.17)$ & $<0.01$ \\
Grip strength & $32.0(8.68)$ & $27.1(7.59)$ & $24.8(8.69)$ & $22.6(8.03)$ & $18.8(7.06)$ & $<0.01$ \\
SPPB & $10.6(250)$ & $9.71(3.10)$ & $9.19(3.21)$ & $8.74(3.32)$ & $7.13(3.80)$ & $<0.01$ \\
\hline
\end{tabular}

Data are expressed mean \pm SD

GNRI, Geriatric Nutritional Risk Index; SPPB, Short Physical Performance Battery

Table 3. Interrelationship between nutritional status and probable sarcopenia index 


\begin{tabular}{|c|c|c|c|c|}
\hline & \multicolumn{4}{|c|}{ Multivariate model } \\
\hline & \multirow{2}{*}{\multicolumn{2}{|c|}{ (man<26 kg, woman<18kg) }} & \multirow{2}{*}{\multicolumn{2}{|c|}{$\begin{array}{l}\text { Loss of SPPB } \\
\text { (<8 points) }\end{array}$}} \\
\hline & & & & \\
\hline & OR $(95 \% \mathrm{CI})$ & $\mathrm{P}$ value & OR $(95 \%$ CI) & $\mathrm{P}$ value \\
\hline $\mathrm{GNRI} \geq 90$ & \multicolumn{2}{|l|}{ Reference } & \multicolumn{2}{|l|}{ Reference } \\
\hline GNRI $<90$ & $2.272(1.636-3.155)$ & $<0.001$ & $1.782(1.303-2.437)$ & $<0.001$ \\
\hline $3.5 \leq \mathrm{P} \leq 6.0(\mathrm{mg} / \mathrm{dL})$ & \multicolumn{2}{|l|}{ Reference } & \multicolumn{2}{|l|}{ Reference } \\
\hline $\mathrm{P} \square 3.5(\mathrm{mg} / \mathrm{dL})$ & $1.400(0.790-2.481)$ & 0.25 & $1.430(0.840-2.435)$ & 0.19 \\
\hline $\mathrm{P} \square 6.0$ (mg/dL) & $1.143(0.760-1.719)$ & 0.52 & $0.632(0.419-0.952)$ & 0.03 \\
\hline $\mathrm{GNRI} \geq 90$ and $3.5 \leq \mathrm{P} \leq 6.0(\mathrm{mg} / \mathrm{dL})$ & \multicolumn{2}{|l|}{ Reference } & \multicolumn{2}{|l|}{ Reference } \\
\hline GNRI $\geq 90$ and $\mathrm{P} \square 3.5(\mathrm{mg} / \mathrm{dL})$ & $0.844(0.357-1.997)$ & 0.70 & $0.588(0.242-1.426)$ & 0.24 \\
\hline GNRI $\geq 90$ and $\mathrm{P} \square 6.0(\mathrm{mg} / \mathrm{dL})$ & $1.099(0.668-1.807)$ & 0.71 & $0.815(0.503-1.322)$ & 0.41 \\
\hline GNRI $\square 90$ and $3.5 \leq \mathrm{P} \leq 6.0(\mathrm{mg} / \mathrm{dL})$ & $2.005(1.345-2.988)$ & 0.001 & $1.690(1.161-2.459)$ & 0.006 \\
\hline GNRI $\square 90$ and $\mathrm{P} \square 3.5$ (mg/dL) & $3.770(1.491-9.533)$ & 0.005 & $6.770(2.658-17.243)$ & $<0.001$ \\
\hline GNRI $\square 90$ and $\mathrm{P} \square 6.0$ (mg/dL) & $2.764(1.309-5.836)$ & 0.008 & $0.663(0.307-1.432)$ & 0.30 \\
\hline
\end{tabular}

The multivariate model was adjusted for sex, dialysis vintage, and age.

SPPB, Short Physical Performance Battery; GNRI, Geriatric Nutritional Risk Index; P, serum phosphorus; OR, Odds ratio; 95\% CI, 95\% confidence interval

\section{Supplementary Files}

This is a list of supplementary files associated with this preprint. Click to download.

- STROBEchecklistcrosssectional.doc 